\title{
Qualidade de rosas de corte tratadas com produtos naturais
}

\author{
Quality of cut roses treated with natural products
}

\author{
Júlia De Pietro ${ }^{\mathrm{I}}$ Ben-Hur Mattiuz ${ }^{\mathrm{I} *}$ Claudia Fabrino Machado Mattiuz ${ }^{\mathrm{I}}$ \\ Teresinha de Jesus Deléo Rodrigues ${ }^{\text {II }}$
}

\section{RESUMO}

\begin{abstract}
O uso de produtos naturais como conservantes florais vem contribuir para a redução do uso indiscriminado de produtos químicos potencialmente tóxicos. Assim, objetivouse neste trabalho testar soluções para manter a qualidade $e$ aumentar a durabilidade comercial de rosas vermelhas cortadas da cv. 'Vega'. Foi empregado o delineamento inteiramente casualizado, em esquema fatorial composto por seis tratamentos pós-colheita e quatro datas de avaliação. Os tratamentos realizados foram os seguintes: 1) Água destilada; 2) Metil jasmonato $(350 \mu M)$; aplicado na forma de pulsing, por quatro horas; 3) Pulverização de Metil jasmonato $(500 \mu \mathrm{M})$; 4) Óleo de menta (100ppm); 5) Óleo de gengibre (100ppm); e 6) Própolis (0,05\%), utilizados na forma de solução de manutenção. As flores foram mantidas em ambiente com temperatura de $20 \pm 2^{\circ} \mathrm{C}$ e umidade relativa de $67 \pm 3 \%$. Procedeu-se a avaliações fisiológicas e qualitativas. De modo geral, os produtos naturais apresentaram ação benéfica sobre a vida de vaso das flores, entretanto, o uso do metil jasmonato em pulverização mostrou, em todos os parâmetros avaliados, ser o produto mais eficiente para manutenção da qualidade floral, promovendo menor perda de massa fresca e menor taxa respiratória das flores, além de permitir melhoria na manutenção da coloração, do conteúdo relativo de água e de carboidratos redutores.
\end{abstract}

Palavras-chave: Rosa sp, metil jasmonato, óleo de menta, óleo de gengibre, própolis.

\section{ABSTRACT}

Use of natural products like floral preservative has contributed to reduce indiscriminate use of chemical products. Therefore, this research tested solutions to keep quality and to increase commercial durability of 'Vega' $c v$. cut roses. It was used a casual design in a factorial schema composed by six post harvest treatments and four evaluation dates. The following treatments were conducted: 1) Distilled water; 2) Metil jasmonate $(350 \mu M)$ application in pulsing form for four hours; 3) Metil jasmonate (500 $\mu$ M) spraying; 4) Mint oil (100ppm); 5) Ginger oil (100ppm); and 6) Propolis (0.05\%) used as a maintenance solution. Flowers stayed in an environment at $20 \pm 2{ }^{\circ} \mathrm{C}$ and $67 \pm 3 \%$ RH. Physiological and qualitative evaluations were conducted. Generally speaking, natural products presented a beneficial action over the shelf life of flowers. However, metil jasmonate spraying was, in all evaluated parameters, the most efficient product to maintain floral quality, providing a lower fresh mass loss and a lower respiratory rate of flowers. It also permitted improvement in coloration maintenance, water relative content and reducing sugars.

Key words: Rosa sp, metil jasmonate, mint oil, ginger oil, propolis.

\section{INTRODUÇÃO}

Uma das principais razões para a perda da qualidade comercial de flores de corte é o bloqueio vascular causado por microrganismos, que inibe o fornecimento de água e nutrientes para as flores. Embora existam poucos estudos relacionados com o uso de produtos naturais como alternativa aos produtos convencionalmente utilizados na fisiologia pós-colheita de flores, antagonistas microbianos naturais têm sido reportados para proteger a vida de

IDepartamento de Tecnologia, Faculdade de Ciências Agrárias e Veterinárias (FCAV), Universidade Estadual de São Paulo (UNESP), Via de Acesso Prof. Paulo Donato Castellane, s/n, 14884-900, Jaboticabal, SP, Brasil. E-mail: benhur@fcav.unesp.br.*Autor para correspondência.

"FCAV, UNESP, Jaboticabal, SP, Brasil 
produtos perecíveis contra um elevado número de patógenos pós-colheita, além de contribuir para a redução do uso indiscriminado de produtos químicos potencialmente tóxicos (WISNIEWSKI et al., 2001).

O ácido jasmônico e seus derivados jasmonatos são reguladores vegetais endógenos produzidos por várias espécies vegetais (LINARES et al., 2010) tais como: Jasminun grandiflorum, Rosmarinus officinalis, Arthemisia absinthium L., sementes imaturas de Phaseolus vulgaris L. eDolicos lablab L. (MEYER et al., 1984). Esses produtos estão relacionados ao mecanismos de defesa vegetal. Eles induzem a expressão de genes que codificam proteínas específicas, como inibidores de proteases, enzimas envolvidas com a produção de flavonoides e diferentes proteínas relacionadas com doenças (CORTÊS, 2000).

Pode ser aplicado de forma eficaz para controlar o fungo Botrytis cinerea (TRIPATHI \& DUBEY, 2004). Opulsing com metil jasmonato controlou a infestação de Botrytis cinerea em rosas (MEIR et al., 2005) e em Chamelaucium uncinatum (DINH et al., 2007). Flores de Paeonia lactiflora tiveram menor incidência de doença e maior longevidade quando tratadas com metil jasmonato(GAST, 2001).

A atividade antifúngica dos óleos essenciais já está bem documentada, existindo estudos de seus efeitos em patógenos pós-colheita (REUVENI et al., 2008). As plantas medicinais e aromáticas como Mentha arvensis L. e Zingiber officinale $\mathrm{R}$., contém princípios ativos antimicrobianos, que podem ser promissores para a conservação de flores de corte, pois atuam no controle de fungos fitopatogênicos, além de não afetarem o meio ambiente (TOSI et al., 1996).

O própolis é uma substância natural resinosa que contém proteínas, aminoácidos, vitaminas, minerais e flavonoides (MOREIRA, 1986). Possui uma atividade antibacteriana e antifúngica e tem sido indicado para inibir patógenos pós-colheita como o Botrytis cinerea e Penicillum expansum.

Assim, avaliou-se a aplicação de produtos naturais, visando à manutenção da qualidade e da durabilidade comercial de rosas cortadas, como alternativa aos produtos químicos que causam danos ao meio ambiente e à saúde humana.

\section{MATERIAL E MÉTODOS}

Foi utilizada a flor de corte Rosa hybrida L., cv. Vega de cor vermelha, cultivada em ambiente protegido e padronizada a $50 \mathrm{~cm}$. Foram estabelecidos os seguintes tratamentos: 1) Água destilada (Controle); 2) Metil jasmonato $95 \%$ (Sigma-Aldrich $®$ ) pulsing $(350 \mu \mathrm{M})$ por quatro horas; 3) Metil jasmonato em pulverização (500 $\mu \mathrm{M})$; 4) Óleo de menta puro (100ppm); 5) Óleo de gengibre puro(100ppm); 6) Extrato de Própolis (Bioessens $^{\odot}$ ) $(0,05 \%)$. Os tratamentos 4, 5 e 6 foram aplicados na forma de solução de manutenção, enquanto que as hastes dos demais tratamentos permaneceram em água destilada.

$\mathrm{O}$ experimento foi conduzido em delineamento inteiramente casualizado, em esquema fatorial composto por dois fatores: seis tratamentos pós-colheita e quatro datas de avaliação $(0,4,8$ e 12 dias). Para cada combinação de fatores, foram utilizadas três repetições com três flores cada uma. Durante o período experimental, as flores foram mantidas em ambiente com temperatura de $20 \pm 2^{\circ} \mathrm{C}$ e umidade relativa de $67 \pm 3 \%$.

Durante as avaliações, foi determinada, por pesagem, a massa fresca das flores. A variação da massa fresca foi calculada em relação à massa inicial.

O conteúdo relativo de água (CRA) das pétalas foi avaliado coletando-se 10 discos com 10mm de diâmetro das pétalas das rosas de cada repetição. Esses discos foram pesados e imersos em água destilada, para hidratação, por 4h. Após esse período, os discos foram novamente pesados e levados para estufa de secagem, a $70^{\circ} \mathrm{C}$ (KRAMER, 1983).

$\mathrm{O}$ teor de $\mathrm{CO}_{2}$ (atividade respiratória) produzido pelas hastes foi quantificado pelo analisador de gases PBI-Dansensor 9900. O teor de carboidratos solúveis e redutores, bem como a coloração das pétalas foram determinados conforme o descrito por (MATTIUZet al., 2010).

As avaliações referentes a escurecimento, turgescência e curvatura das hastes foram efetuadas utilizando-se critério de notas: escurecimento das pétalas: nota $4=$ nenhuma pétala escurecida; nota $3=5$ a $19 \%$ de pétalas escurecidas; nota $2=20$ a $29 \%$; nota $1=30 \%$ ou valor acima; turgescência: nota $4=$ túrgida; nota 3 = levemente murcha; nota $2=$ murcha; nota $1=$ totalmente murcha; curvatura da flor em relação ao pedúnculo: nota 4 = flor reta; nota $3=$ ângulo entre $1 \mathrm{e}$ $30^{\circ}$; nota 2 a ângulo entre 31 e $90^{\circ}$; nota $1=$ ângulo maior que $90^{\circ}$ (considerado descarte comercial).

Os dados obtidos foram submetidos à análise de variância e as médias foram comparadas pelo teste de diferença mínima significativa em teste de comparações múltiplas. Assim, as diferenças entre dois tratamentos, quando maior que a soma de dois desvios padrões, foram consideradas significativas em nível de 5\% de probabilidade (MATTIUZet al., 2010). Para a longevidade, foi feita análise de variância pelo teste $\mathrm{F}$ e as médias foram comparadas utilizando o teste de Tukey $(\mathrm{P} \leq 0,05)$. 


\section{RESULTADOS E DISCUSSÃO}

A perda de massa fresca aumentou ao longo do período de armazenamento, principalmente entre os dias seis e nove, em todos os tratamentos, conforme o mostrado na figura $1 \mathrm{~A}$. Porém, a intensidade dessa perda foi mais acentuada no tratamento Controle (água destilada) quando comparado aos outros tratamentos. A perda de massa pode ser causada pela transpiração, pela diminuição na absorção de água devido à obstrução da haste por microrganismos ou por fatores geneticamente determinados. ICHIMURA et al. (2006) sugerem que a longevidade floral está diretamente relacionada à manutenção de um nível elevado, ou pelo menos constante, da massa fresca das flores durante a vida pós-colheita.

Salienta-se que a pulverização com metil jasmonato foi o tratamento que se destacou pela menor perda acumulada de massa fresca $(14,67 \%)$, assim como observado por DARRAS et al. (2005) em Freesia hybrida L. Nesse caso, a absorção de água pode ter sido favorecida devido à regulação de vários aspectos de desenvolvimento das plantas, proporcionada pelo metil jasmonato, tal como os mecanismos de defesa microbiana.

O conteúdo relativo de água diminuiu no decorrer do período experimental e em todos os tratamentos (Figura 1B). A água destilada, simplesmente, não foi suficiente para manter a turgidez das pétalas, visto que apresentou a maior redução do conteúdo relativo de água, diferindo significativamente dos demais tratamentos, com exceção do própolis. A perda de água ou o estresse hídrico, durante os dias de vida no vaso, inicia-se quando o conteúdo de água nos tecidos intracelulares se afasta do ótimo.

Todavia, o tratamento de pulverização com metil jasmonato exerceu efeito positivo no balanço hídrico das pétalas, apresentando a menor redução do conteúdo relativo de água, de forma que conferiu maior turgidez às flores (Figura 1B). Esses resultados são

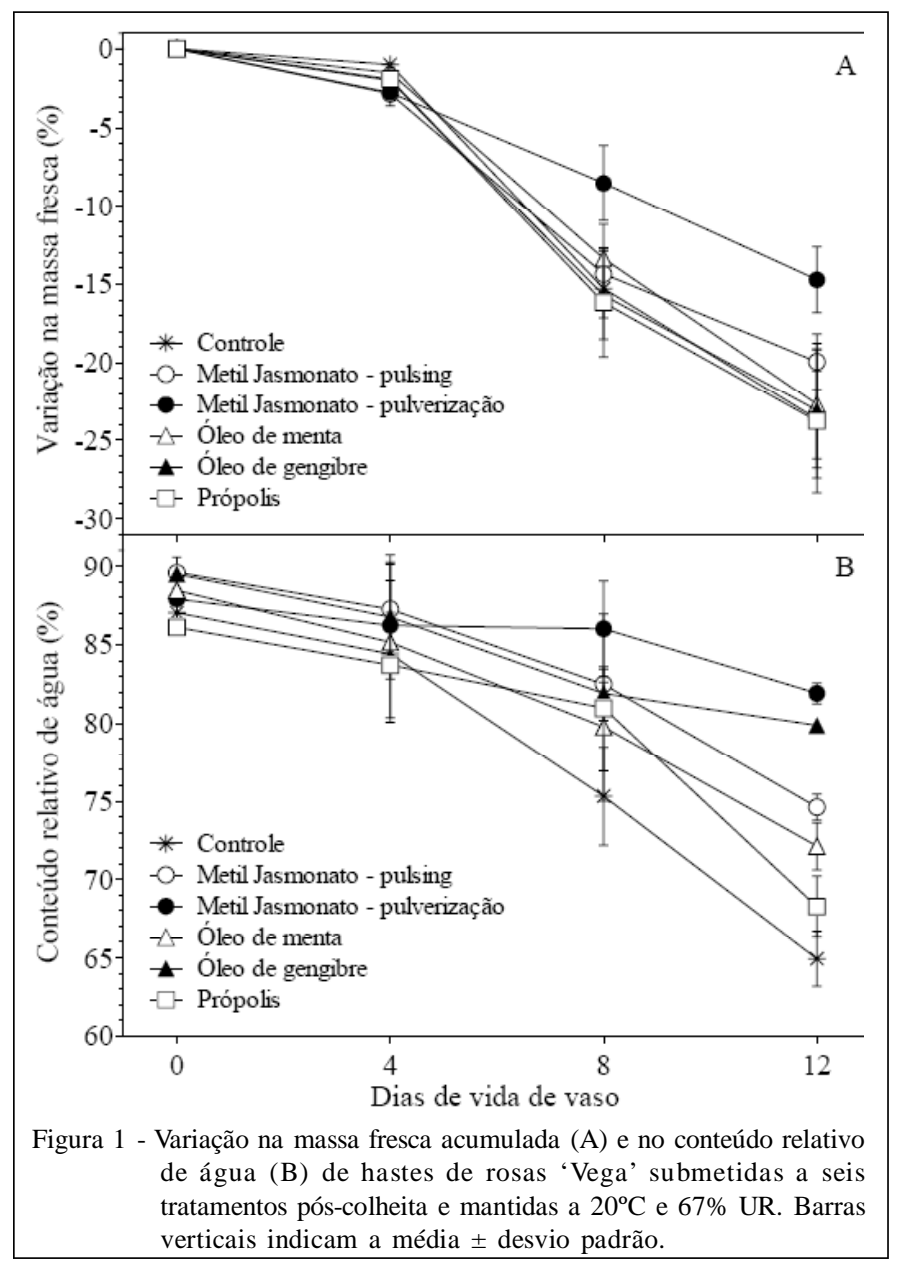

Ciência Rural, v.42, n.10, out, 2012. 
coerentes com os encontrados por CORTES et al. (2011) em rosas cv. 'Grand Gala', os quais afirmam que altos níveis de hidratação dos tecidos são, em geral, associados ao aumento da vida de vaso das flores de corte.

Sabe-se que a menor taxa respiratória favorece o aumento da vida de vaso que, neste caso, é caracterizado principalmente pelo menor uso de reservas como substrato respiratório e, por sua vez, gera energia vital a elas, influenciando positivamente as características qualitativas das flores. Esse efeito foi conseguido pelo tratamento de pulverização com metil jasmonato (Figura 2A). Ainda, observa-se que o tratamento com água destilada apresentou a maior taxa respiratória, e coincidiu com os resultados de maior perda acumulada de massa fresca (Figura 1A), possivelmente em decorrência da utilização apenas de reservas próprias para a manutenção do metabolismo. Isso é suportado por HARDENBURG et al. (1990) ao argumentar que as flores de corte têm uma vida muito limitada depois de destacadas da planta mãe, pois sobrevivem com suas próprias reservas, que são geralmente reduzidas, por causa das próprias características morfológicas e fisiológicas dos tecidos.

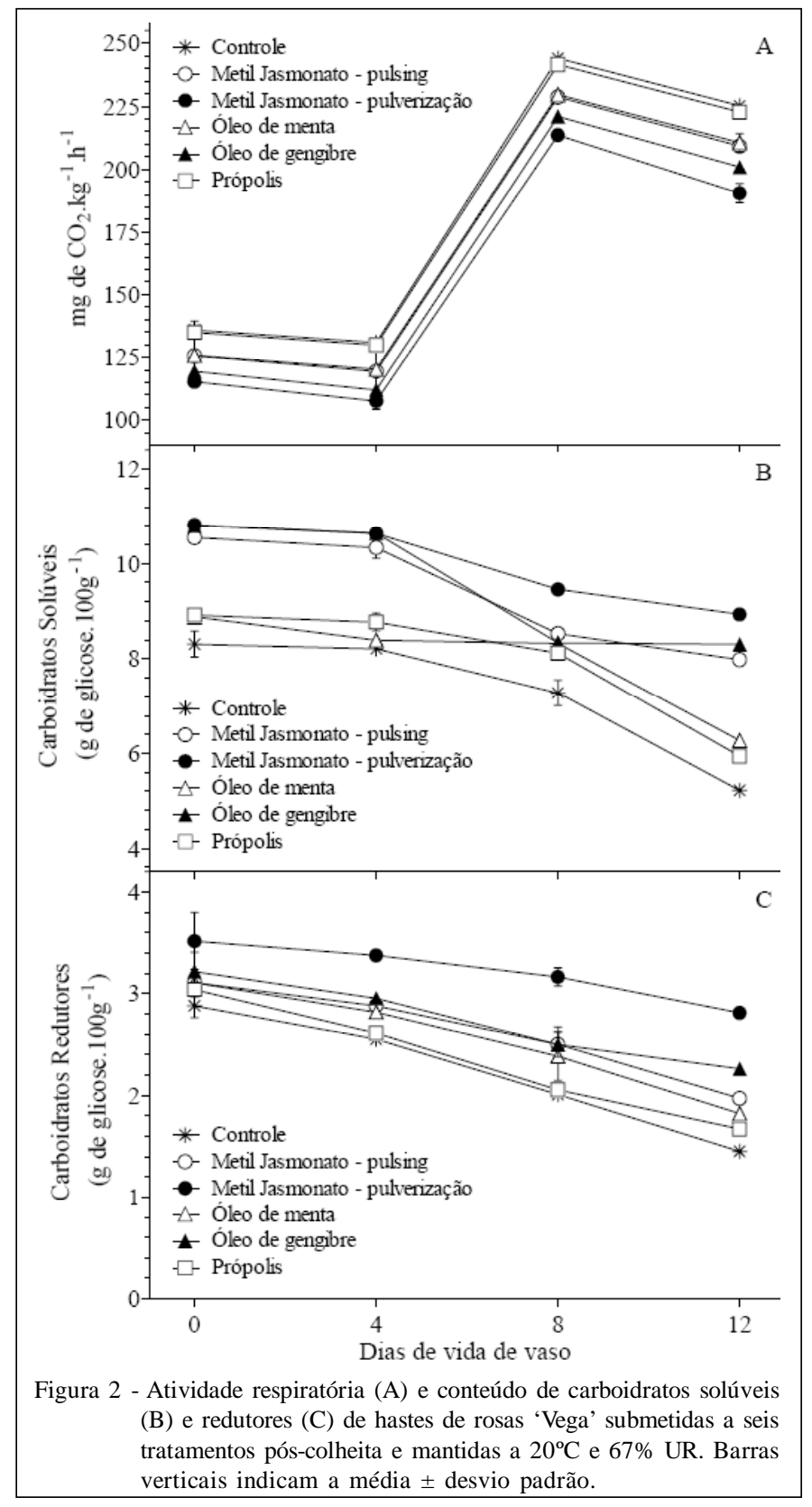

Ciência Rural, v.42, n.10, out, 2012. 
Ocorreu redução significativa no conteúdo dos carboidratos solúveis (Figura 2B) e redutores (Figura 2C) para todos os tratamentos. No entanto, o tratamento de pulverização com metil jasmonato expressou a menor utilização das reservas orgânicas, já que o decréscimo nas quantidades de carboidratos solúveis e redutores foram menores se comparadas com os demais tratamentos. A maior redução dos teores de carboidratos solúveis e redutores foi detectada no tratamento com água destilada, embora este não tenha diferido significativamente das flores tratadas com solução de própolis.

Esses resultados podem ser relacionados com a taxa respiratória (Figura 2A), visto que a maior redução dos carboidratos solúveis e redutores acompanhou a maior atividade respiratória, ou seja, houve um maior gasto de energia que reduziu a longevidade dos órgãos das flores. TAIZ \& ZEIGER (2004) relataram que, para a maioria das plantas, a sacarose é o principal açúcar translocado e, portanto, a forma de carbono que a maioria dos tecidos não fotossintéticos importa, podendo ser considerada como principal substrato para a respiração vegetal.

O uso do metil jasmonato em pulverização destacou-se dos demais tratamentos, com as melhores notas atribuídas ao escurecimento, turgescência e curvatura (Figura 3). Quanto à cor, este trabalho propõe que o metil jasmonato deve atuar no metabolismo das

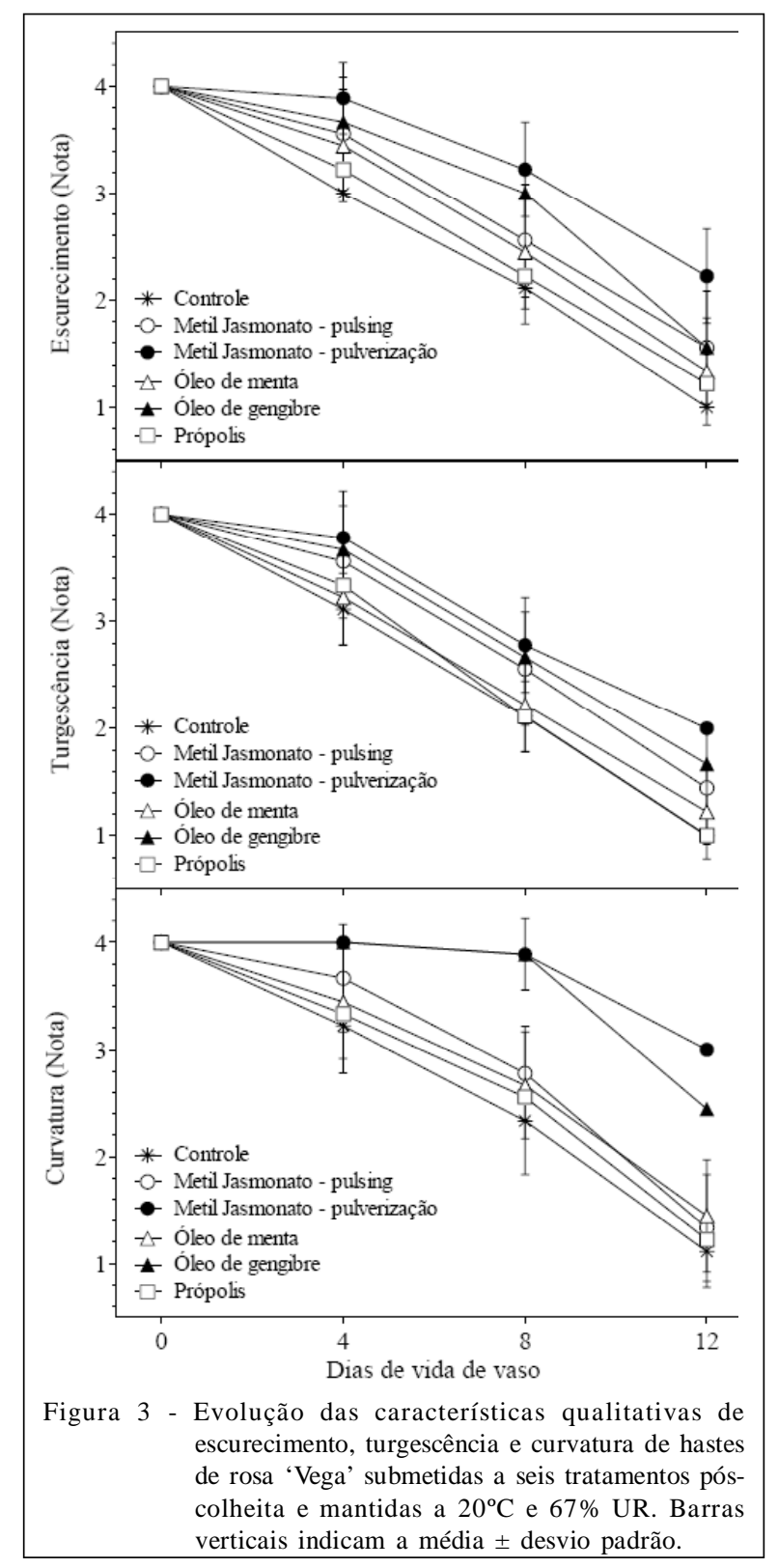

Ciência Rural, v.42, n.10, out, 2012. 
antocianinas evitando o enegrecimento das pétalas. Provavelmente, a menor curvatura da haste foi favorecida pela diminuição de microrganismos na solução de vaso, uma vez que, segundo pesquisa com seis cultivares de rosas realizada por MEIR et al. (2005), o metil jasmonato é capaz de fornecer proteção sistêmica ou local contra o Botrytis cinerea. Contudo, as hastes imersas em água destilada obtiveram as menores notas, confirmando juntamente com resultado de perda de massa fresca que este foi o tratamento que proporcionou menor manutenção de qualidade às hastes de rosa da cv. Vega, caracterizada, neste caso, pela desestabilização da cor vermelha, perda de água e curvatura.

A figura 4 apresenta os resultados referentes à coloração das pétalas. Observa-se que houve maior redução da luminosidade no tratamento com água destilada, indicando uma tendência ao escurecimento das pétalas, enquanto a melhor manutenção foi observada pelo tratamento de pulverização com metil jasmonato, que teve comportamento semelhante ao óleo de gengibre. O escurecimento é causado pela oxidação de fenóis, principalmente as leuco-antocianinas, no entanto, REID (2002) constatou escurecimento em

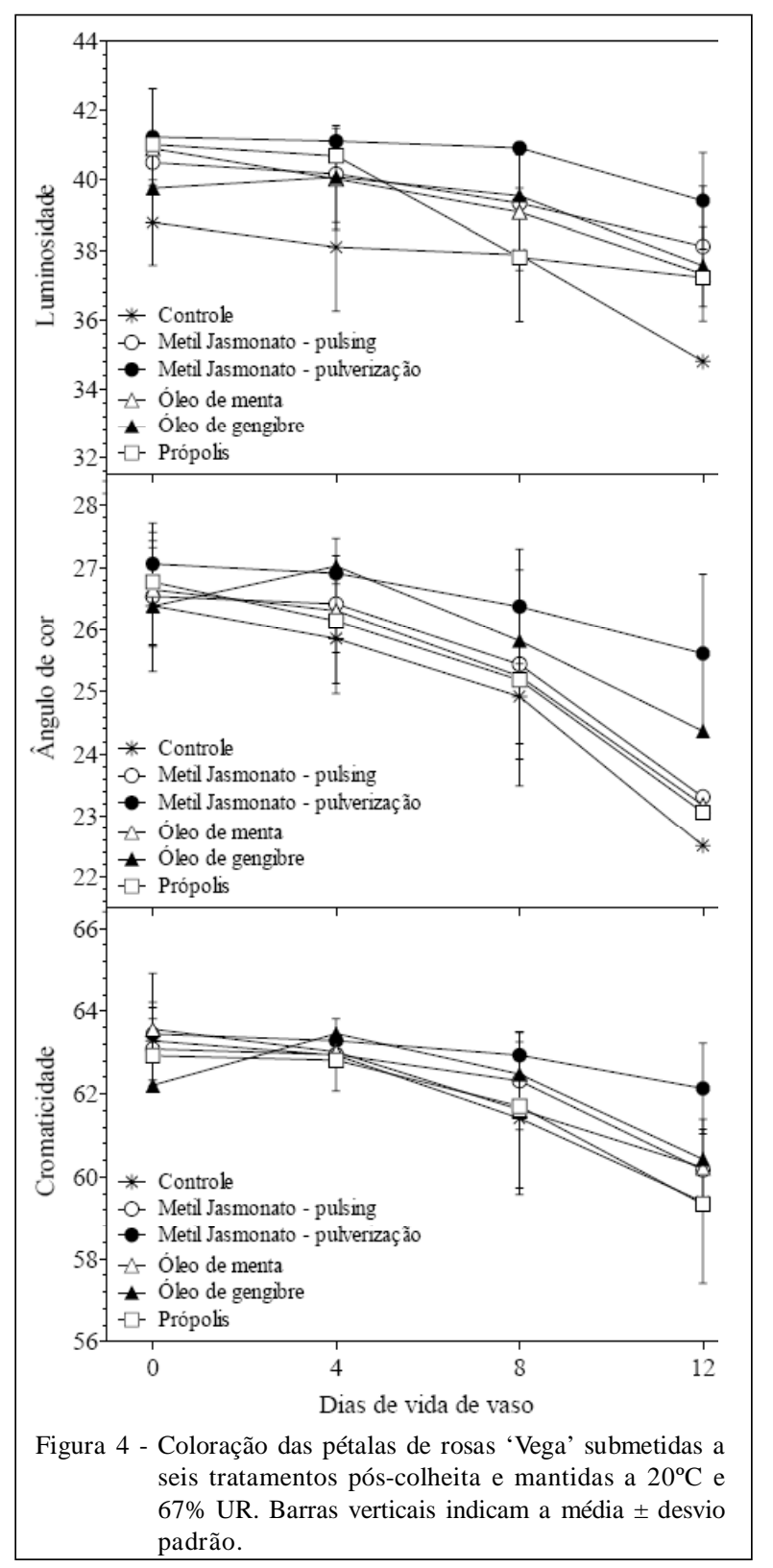

Ciência Rural, v.42, n.10, out, 2012. 
Protea nerifolia e foi atribuído ao baixo nível de carboidratos, por ocasião da colheita.

A melhor manutenção da cor vermelha, expressa pelo ângulo de cor, foi obtida no tratamento de pulverização com metil jasmonato, enquanto as flores tratadas com água destilada expressaram a maior redução desse parâmetro. Para a cromaticidade, notase que o tratamento em água destilada sofreu a maior redução durante os dias de vida de vaso e o tratamento de pulverização com metil jasmonato manteve a melhor intensidade, sendo semelhante aos resultados obtidos para o tratamento com óleo de gengibre. Para CHITARRA \& CHITARRA (2005), a coloração é o atributo de qualidade mais atrativo para o consumidor, sendo os produtos de cor forte e brilhante os preferidos. Denota-se que houve redução da luminosidade, ângulo de cor e cromaticidade em todos os tratamentos (Figura 4).

Observa-se, na tabela 1 , que a pulverização com metil jasmonato diferiu significativamente das demais soluções testadas, em função da maior longevidade (12 dias). Resultados semelhantes foram obtidos por MEIR et al. (2005), que constataram que o uso do metil jasmonato na forma de pulsing $(350 \mu \mathrm{M}) \mathrm{e}$ em pulverização na concentração de $500 \mu \mathrm{M}$ suprimiu a infestação de Botrytis cinerea em rosas de corte. Os jasmonatos são reguladores de crescimento naturais e são conhecidos por regularem vários aspectos do desenvolvimento das plantas e por responderem a estresses ambientais; a eles também tem sido atribuída a capacidade de transcrição de muitos genes envolvidos no metabolismo de defesa vegetal (TAIZ \& ZEIGER, 2004). Tais efeitos merecem ser mais investigados, pois sua ação antimicrobiana poderá ampliar o seu uso e ser uma alternativa viável aos tratamentos com compostos químicos potencialmente potenciais.

Tabela 1 - Longevidade, expressa em dias de vida de vaso, de hastes de rosas, submetidas a seis tratamentos póscolheita e mantidas a $20^{\circ} \mathrm{C}$ e $67 \%$ UR.

\begin{tabular}{lc}
\hline Tratamentos & Longevidade \\
\hline Controle & $9,00 \mathrm{~d} \alpha$ \\
Metil Jasmonato - pulsing & $11,00 \mathrm{~b}$ \\
Metil Jasmonato - pulverização & $12,00 \mathrm{a}$ \\
Óleo de menta & $10,00 \mathrm{c}$ \\
Óleo de gengibre & $11,00 \mathrm{~b}$ \\
Própolis & $9,00 \mathrm{~d}$ \\
& \\
$\mathrm{CV}(\%)$ & 12,0 \\
\hline
\end{tabular}

$\alpha$ Médias seguidas de pelo menos uma letra em comum na coluna não diferem significativamente entre si pelo teste de Tukey $(\mathrm{P}<0,05)$.

\section{CONCLUSÃO}

A aplicação de metil jasmonato por pulverização em rosa vermelha cv. Vega se destacou dos demais produtos naturais, possibilitando uma maior longevidade média das flores e melhor manutenção das características comerciais desejáveis.

\section{AGRADECIMENTO}

À Fundação de Amparo à Pesquisa do Estado de São Paulo (FAPESP), que concedeu uma bolsa de Mestrado (Proc. 2007/57918-9) para a realização desta pesquisa.

\section{REFERÊNCIAS}

CHITARRA, M.I.F.; CHITARRA, A.B. Pós-colheita de frutos e hortaliças: fisiologia e manuseio. Lavras: ESAL, 2005. $785 \mathrm{p}$.

CORTES, M.H. et al. The effects of calcium on postharvest water status and vase life of Rosa hybrida cv. Grand Gala. International Journal of Agriculture and Biology, v.13, n.2, p.233-238, 2011. Disponível em: <http:// www.fspublishers.org/ijab/past-issues/IJABVOL_13_NO_2/ 14.pdf>. Acesso em: nov. 2011. doi: 10-027/SBC/2011/132-233-238.

CORTÊS, H.P. Jasmonatos. In: CID, L.P.B. Introdução aos hormônios vegetais. Brasília: EMBRAPA, 2000. p.131157 .

DARRAS, A.I. et al. Methyl jasmonate vapour treatment suppresses specking caused by Botrytis cinerea on cut Freesia hybrida L. flower. Postharvest Biology and Technology, v.38, n.2, p.175-182, 2005. Disponível em:<http://dx.doi.org/ 1016/j.postharvbio.2005.06.011>. Acesso em: jan. 2011. doi: $10.1016 / \mathrm{j}$.postharvbio.2005.06.011.

DINH, S.Q. et al. Effects of combined methyl jasmonate and ethylene-inhibitor treatments against Botrytis cinerea infecting Geraldton Waxflower. Acta Horticulturae, v.1, n.755, p.527533, 2007.

GAST, K. Methyl jasmonate and long term storage of fresh cut peony flowers. Acta Horticulturae, v.1, n.543, p.327-330, 2001 .

HARDENBURG, R.E. et al. The commercial storage of fruits, vegetables and florist and nursery stocks. Washington: U.S.D.A, Agricultural Research Service, 1990. 130 p.

ICHIMURA, K. et al. Effects of temperature, 8hydroxyquinoline sulphate and sucrose on the vase life of cut rose flowers. Postharvest Biology and Technology, v.5, p.33-34, 2006. Disponível em: <http://dx.doi.org/10.1016/ SO925-5214(98)00063-5>. Acesso em: jan. 2011. doi: 10.1016/SO925-5214(98)00063-5.

KRAMER, P.J. Water relations of plants. New York: Academic, 1983. 489p. 
LINARES, A.M.P. et al. Atividade fitorreguladora de jasmonatos produzidos por Botryosphaeria rhodina. Horticultura Brasileira, v.28, n.4, p.430-434, 2010. Disponível em: <http://dx.doi.org/10.1590/S0102-05362010000400009>. Acesso em: fev. 2011. doi: 10.1590/S010205362010000400009 .

MATTIUZ, C.F.M. et al. Armazenamento refrigerado de inflorescências cortadas de Oncidium varicosum 'Samurai'. Ciência Rural, v.40, n.11, p.2288-2293, 2010. Disponível em: <http://dx.doi.org/10.1590/S0103-84782012005000028>. Acesso em: jan. 2011. doi: 10.1590/S010384782012005000028 .

MEIR, S. et al. Use of methyl jasmonate for suppression of Botrytis rot in various cultivares of cut rose flowers. Acta Horticulturae, v.1, n.669, p.91-98, 2005.

MEYER, A. et al. Occurrence of the plant growth regulators jasmonic acid in plants. Journal of Plant Growth Regulator, v.3, p.1-8, 1984. Disponível em: <https:// springerlink3.metapress.com/content/v10577n1r51330n5/ resource-secured $/$ target $=$ fulltext.pdf $\&$ sid $=\mathrm{kpzmi} 24 \mathrm{vx} 3$ 3 wlfzi1 mloe0by\&sh=www.springerlink.com>. Acesso em: jan. 2011. doi: 10.1007/BF02041987.

MOREIRA, T.E. Chemical composition of propolis, vitamins and amino acids. Revista Brasileira de Farmacologia, v.1, n.1, p.12-19, 1986.
REID, M.S. Postharvest handling systems: ornamental crops. In: KADER, A.A. Postharvest technology of horticultural crops. Davis: University of California, 2002. p.315-326.

REUVENI, R. et al. Fungistatic activity of essential oils from Ocimum basilicum Chemotypes. Journal of Phytopathology, v.110, n.1, p.20-22, 2008. Disponível em: <http://dx.doi.org/ 10.1111/j.1439-0434.1984.tb00736.x>. Acesso em: jan. 2011. doi: 10.1111/j.1439-0434.1984.tb00736.x.

TAIZ, L.; ZEIGER, E. Fisiologia vegetal. Porto Alegre: Artmed, 2004. 719p.

TOSI, B. et al. Antimicrobial activity of some commercial extracts of propolis prepared with different solvents. Phytotherapy Research, v.10, n.4, p.335-336, 1996.

TRIPATHI, P; DUBEY, N.K. Exploitation of natural products as an alternative strategy to control postharvest fungal rotting of fruit and vegetables. Postharvest Biology and Technology, v.31, n.3, p.235-245, 2004. Disponível em: <http://dx.doi.org/ 10.1016/j.postharvbio.2003.11.005>. Acesso em: jan. 2011. doi: $10.1016 /$ j.postharvbio.2003.11.005.

WISNIEWSKI, M. et al. Non chemical approaches to postharvest disease control. Acta Horticulturae, v.1, n.553, p.407-412, 2001. 